\title{
Antenatal diagnosis of inborn errors of metabolism
}

\author{
M A Cleary, J E Wraith
}

The introduction of experimental treatment for lysosomal storage disorders and the increasing understanding of the molecular defects behind many inborn errors have overshadowed the fact that for many affected families the best that can be offered is a rapid, accurate prenatal diagnostic service. Many conditions remain at best only partially treatable and as a consequence the majority of parents seek antenatal diagnosis in subsequent pregnancies, particularly for those disorders resulting in a poor prognosis in terms of either life expectancy or normal neurological development.

The majority of inborn errors result from a specific enzyme deficiency, but in some the primary defect is in a transport system or enzyme cofactor. In some conditions the biochemical defect is limited to specific tissues only and this serves to restrict the material available for antenatal diagnosis for these disorders. Fortunately for many inborn errors the metabolic defect is generalised and both amniotic and chorion villus cells can be used as a diagnostic tissue.

Before contemplating prenatal diagnosis it is essential that a firm biochemical diagnosis has been established in the index case. It is unsafe to rely only on a clinical or histological diagnosis as many of the inherited disorders share a similar phenotype. To ease interpretation of results obtained on the fetus it is necessary to know the heterozygote levels of enzyme activity in the parents; occasionally these are remarkably low and can lead to difficulties in ascribing fetal genotype.

The properties of some enzymes are appreciably different when studied at different times of gestation. In addition, the activity obtained from chorion villus material may be very different from that obtained on amniotic fluid cells. It is essential that the correct tissue is collected at the most appropriate time. Liaison with the laboratory staff performing the test is mandatory if mistakes are to be avoided.

Prenatal testing by analysis of fetal DNA by either a gene specific DNA probe or gene tracking using restriction fragment length polymorphisms (RFLPs) requires proband DNA for comparison. This underlies the importance of establishing fibroblast cultures from all patients diagnosed as having a metabolic disorder as well as ensuring that blood is taken from all relevant family members for DNA extraction and storage.

Most prenatal testing for metabolic disease is performed in a few specialised laboratories.

\section{Sample requirement and techniques used in} prenatal diagnosis

By far the majority of antenatal diagnoses are performed on samples obtained by either amniocentesis or chorion villus biopsy. For some disorders, however, the defect is not detectable in this material and more invasive methods have been applied to obtain a diagnostic sample.

\section{FETAL LIVER BIOPSY}

Fetal liver biopsy has been performed to diagnose ornithine carbamoyl transferase deficiency and primary hyperoxaluria type 1 . Glucose-6-phosphatase deficiency (glycogen storage disease type I) could also be detected by this method. The technique, however, is invasive and can be performed by only a few highly specialised fetal diagnostic units.

\section{FETAL BLOOD SAMPLING}

Fetal blood sampling could be used for the antenatal diagnosis of many inborn errors. The sample, however, tends to be collected late in pregnancy and the technique is probably best reserved as a back up in case of failed amniotic fluid cell culture.

\section{AMNIOCENTESIS}

Amniocentesis has been the most common procedure for antenatal diagnosis of metabolic disease. Both the cell free amniotic fluid and cultured and uncultured amniotic fluid cells are useful in diagnosis.

The procedure is usually performed at $15-16$ weeks' gestation and most analyses can be performed within two to three weeks of culture. First trimester amniocentesis has been attempted to allow earlier diagnosis, but the smaller sample size and possible variation in enzyme activity at the earlier stage of pregnancy have introduced variables that need to be studied before the technique can be widely applied.

(1) Cell free amniotic fluid can be used to detect a number of intermediary metabolites in many inborn errors. Where possible this technique should be backed by specific enzyme analysis, but for many conditions does allow a quick diagnosis. It is particularily relevant for organic acid disorders using stable isotope dilution gas chromatography with mass spectrometry and selected ion monitoring.

In mucopolysaccharide disorders the pattern 
of excess glycosaminoglycan excretion can be detected by two dimensional electrophoresis and this can form the basis of a reliable antenatal test for this group of conditions.

(2) Amniotic fluid cells in culture are a common material used in antenatal diagnosis. In most cases the enzyme activity obtained in this tissue mirrors that obtained in cultured skin fibroblasts. As well as direct enzyme assay, radiolabelled incorporation studies can be performed on the cultured cells and a wide range of disorders detected. Contamination by mycoplasma or other organisms is the biggest threat to antenatal diagnosis by this method.

CHORION VILLUS BIOPSY

Chorion villus biopsy offers the advantage of first trimester diagnosis. Direct analysis of enzyme activity on fresh uncultured villus tissue usually allows a result to be available within 24 48 hours of the procedure. Problems associated with the technique include the possibility of maternal contamination and differences in enzyme activity in this tissue as compared with skin fibroblasts or cultured amniotic fluid cells. Despite these possible limitations most prenatal diagnoses for inborn errors are performed on chorion villus samples.

\section{Specific inborn errors of metabolism}

The appendix lists the specific inborn error, the biochemical defect, chromosome location of the gene mutation where known, and the test used for antenatal diagnosis. Where antenatal diagnosis has been successfully achieved the method used is clearly shown. For some disorders prenatal diagnosis is theoretically possible, although to the authors' knowledge not as yet successfully performed. In this case the most likely tissue and method are indicated with the rider, 'possible'. The key to the abbreviations used is shown at the end of the appendix.

\section{Appendix}

Specific inborn errors of metabolism

Condition

(A) CARBOHYDRATE METABOLISM

(i) Galactose

Galactokinase deficiency

'Classical' galactosaemia

Epimerase deficiency

(ii) Fructose

Hereditary fructose intolerance

Fructose 1-6 bisphosphatase deficiency

(iii) Glycogen storage disease

Ia Von Gierke

b

II Pompe

III Debrancher enzyme deficiency

IV Brancher enzyme deficiency

V McCardle

VI Liver phosphorylase deficiency

VII Phosphofructokinase deficiency

IXa Phosphorylase kinase (recessive)

IXb Phosphorylase kinase (X linked)

(B) AMINO ACID METABOLISM

(i) Phenylalanine

'Classical' phenylketonuria

Tetrabiopterin homoeostasis

Tetrabiopterin synthesis
Enzyme deficiency

Galactokinase

Galactose-1-phosphateuridyltransferase

UDP-Galactose-4-epimerase

Aldolase B

Frutose $1-6$ bisphosphatase

$9 q 22$

17q21-q22

9 p13

1pter-p32

AF, CVB

Poss CC

Glucose-6-phosphatase

Glucose-6-phosphatase

translocase $\left(T_{1}\right)$

Glucose-6-phosphatase translocase $\left(\mathrm{T}_{2}\right)$

Lysosomal acid glucosidase

Amylo-1-6-glucosidase

1-4-Glucan 6-glycosyltransferase

Muscle phosphorylase

Liver phosphorylase

Phosphofructokinase

Phosphorylase kinase

Phosphorylase kinase

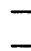

-

-

$17 \mathrm{q} 23$

-

$-$

1lql3-qter

14

1cen-q32

$16 \mathrm{q} 12-\mathrm{q} 13 \cdot 1$

Xq12-q13

Poss DNA

No

Poss FT

Poss FT

Poss FT

AF, CVB

AF, CVB

AF, CVB

No

Poss FT

No

Poss FT

Poss FT
Phenylalanine hydroxylase

Dihydropteridine reductase Guanosine triphosphate cyclohydrolase

6-Pyruvoyltetrahydropterin synthase

$\begin{array}{ll}12 q 22-q 24 \cdot 1 & \text { DNA } \\ 4 p 15 \cdot 3 & \text { AF, CVB } \\ - & \text { MET }\end{array}$

MET 
Condition

(ii) Methionine

Homocystinuria

(iii) Tyrosine

Tyrosinaemia I

Tyrosinaemia II

(iv) Valine, leucine, isoleucine Maple syrup urine disease

(v) Glycine

Non-ketotic hyperglycinaemia

(vi) Lysine

Hyperlysinaemia

(vii) Proline

Hyperprolinaemia I

Hyperprolinaemia II

Hyperimidodipeptiduria

(viii) Ornithine

Gyrate atrophy of the choroid and retina

Hyperornithinaemiahyperammonaemiahomocitrullinaemia $(\mathrm{HHH}$ syndrome)

(C) UREA CYCLE DISORDERS

$\mathrm{N}$-acetylglutamate synthetase deficiency

Carbamyl phosphate synthetase deficiency (CPS)

Ornithine carbamyltransferase deficiency (OCT)

Citrullinaemia

Argininosuccinic aciduria (ASA)

Argininaemia

D) ORGANIC ACID DISORDERS

(i) Propionate and methylmalonate metabolism

Propionic acidaemia

Multiple carboxylase deficiency

Methylmalonic acidaemia

Pyruvate carboxylase deficiency

Phosphoenolpyruvate carboxykinase deficiency

cblA

cblB

Pyruvate dehydrogenase deficiency
Enzyme deficiency

Cystathionine synthase

Chromosome

Prenatal

location

diagnosis

Fumarylacetoacetate hydrolase

Tyrosine aminotransferase

$21 \mathrm{q} 22-\mathrm{q} 22 \cdot 1$

AF, CVB

Branched chain ketoacid dehydrogenase

Glycine cleavage system

$9 \mathrm{p} 22$

CVB

Aminoadipic semialdehyde synthase

Proline oxidase

Pyrroline-5-carboxylate dehydrogenase

Prolidase

Ornithine aminotransferase

$15 q 23-q 25$

$16 \mathrm{q} 22-\mathrm{q} 22 \cdot 1$

AF, CVB

No

19q13·1-q13·2 AF, CVB

Basic defect unknown

$\mathrm{N}$-acetylglutamate synthetase

Carbamyl phosphate synthetase $2 p$

Ornithine carbamyltransferase

$\mathrm{Xp} 21 \cdot 1$

Argininosuccinic acid synthetase

Argininosuccinate lyase

Arginase

9q34

$7 \mathrm{p} 21$-cen

$6 \mathrm{q} 23$

UNK

Poss DNA

FT

Poss DNA

FT

$\mathrm{AF}, \mathrm{CVB}$

AF, CVB

Poss DNA/

FT

Propionyl-CoA carboxylase:

$\alpha$-subunit

$\beta$-subunit

Holocarboxylase synthetase

Biotinidase

Methylmalonyl-CoA mutase

Adenosylcobalamin synthesis:

(see also cobalamin metabolism)

13

$3 q 13 \cdot 3-q 22$

AF, CVB

$-$

$6 \mathrm{p} 12-\mathrm{p} 21 \cdot 2$

AF, CVB

AF, CVB

AF, CVB

Lactate dehydrogenase

$11 \mathrm{p} 15 \cdot 4$

UNK

Pyruvate dehydrogenase complex:

$$
\begin{aligned}
& \mathrm{E}_{1} \text { (decarboxylase) } \\
& \text { component }
\end{aligned}
$$

$\alpha-\mathrm{Xp} 22 \cdot 1-22 \cdot 2$

Poss DNA

$\mathrm{E}_{2}$ (dihydrolipoyl transacylase)

$\beta-3 p 13-q 23$

$\mathrm{E}_{3}$ (dihydrolipoyl dehydrogenase) $7 \mathrm{p} 15-\mathrm{q} 35$

Pyruvate dehydrogenase phosphatase

Pyruvate carboxylase

Phosphoenolpyruvate carboxykinase
AF

UNK 
(iii) Respiratory transport chain defects

The components of the respiratory transport chain are composed of many polypeptide subunits some of which are encoded by mitochondrial DNA
Complex I
Complex II
Complex III
NADH CoQ reductase
Succinate CoA reductase
Complex IV
$\mathrm{CoQH}_{2}$-cyt c reductase
Complex V
Cytochrome oxidase
Oligomycine sensitive ATPase

(iv) Branched chain organic acidaemias

\section{Isovaleric acidaemia \\ Isolated 3-methyl crotonyl CoA carboxylase deficiency \\ 3-Methylglutaconic aciduria \\ 3-Hydroxy-3-methylglutaryl \\ CoA lyase deficiency \\ Mevalonic aciduria \\ 2-Methylacetoacetyl-CoA \\ thiolase deficiency \\ (v) Disorders of the $\gamma$-glutamyl cycle \\ 5-Oxoprolinuria \\ $\gamma$-Glutamylcysteine synthetase \\ deficiency \\ $\gamma$-Glutamylcysteine synthetase deficiency \\ 5-Oxoprolinase deficiency}

Isovaleryl-CoA dehydrogenase

3-Methylcrotonyl carboxylase

$$
15
$$

3-Methylglutaconic hydratase

3-Hydroxy-3-methylglutaryl-

CoA lyase

Mevalonate

2-Methylacetoacetyl-CoA thiolase

$\begin{array}{ll}\text { 15q14-q15 } & \text { MET, AF } \\ - & \text { Poss CC } \\ \overline{\text { AF }, \text { CVB }} & \text { Poss CC } \\ - & \\ - & \text { AF } \\ - & \text { Poss CC }\end{array}$

$\gamma$-Glutamylcysteine synt

$\gamma$-Glutamyltranspeptidase

-

Poss CC

Poss CC

Poss CC

5-Oxoprolinase

Poss CC

(vi) Other organic acid disorders

Alkaptonuria

Glutaric aciduria type I

Glutaric aciduria type II

Glycerol kinase deficiency

Hyperoxaluria type I (glycolic aciduria)

Hyperoxaluria type II (glyceric aciduria)

Canavan's disease

(E) FATTY ACID OXIDATION DEFECTS

Short chain acyl-CoA dehydrogenase deficiency (SCAD)

Medium chain acyl-CoA dehydrogenase deficiency (MCAD)

Long chain acyl-CoA dehydrogenase deficiency (LCAD)

(F) LYSOSOMAL ENZYME DEFECTS

(i) Mucopolysaccharidoses

Type IH (Hurler's syndrome)

Type IS (Scheie's syndrome)

Type II (Hunter's syndrome)

Type III (Sanfilippo's syndrome)

A
B
C

D

Type IV (Morquio's syndrome) A

Type VI (Maroteaux-Lamy syndrome)

Type VII (Sly's disease)
Short chain acyl-CoA

dehydrogenase

12q22-qter

Poss CC

Medium chain acyl-CoA dehydrogenase

1 p31

DNA/CVB

Long chain acyl-CoA dehydrogenase

Poss CC
Iduronidase

Iduronidase

Iduronate sulphatase

Heparan N-sulphatase

$\mathrm{N}$-acetylglucosaminidase

Acetyl-CoA-glucosaminide acetyltransferase

$\mathrm{N}$-acetylglucosamine 6-sulphatase $12 \mathrm{q} 14$

Galactosamine 6-sulphatase

$\beta$-Galactosidase

$\mathrm{N}$-acetylgalactosamine

4-sulphatase

$\beta$-Glucuronidase
$4 \mathrm{p} 16 \cdot 3$

Xq28

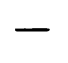

一

3p21-cen

5q11-q13

$7 \mathrm{q} 11 \cdot 2-q 22$
AF, CVB

AF, CVB

AF, CVB

$\mathrm{AF}, \mathrm{CVB}$

AF, CVB

Poss CC

Poss CC

AF, CVB

Poss CC 
Condition

Enzyme deficiency

(ii) Mucolipidoses

Mucolipidosis II (I-Cell disease)

Mucolipidosis III (pseudoHurler polydystrophy)

(iii) Glycoproteinoses

$\alpha$-Mannosidosis

$\beta$-Mannosidosis

Fucosidosis

Aspartylglycosaminuria

Sialidosis type I (cherry-red spot-myoclonus syndrome)

Sialidosis type II:

Congenital and infantile

Juvenile

(iv) $G m_{2}$ gangliosidoses

Tay-Sach's disease (variant B)

Sandhoff's disease (variant 0)

$\mathrm{Gm}_{2}$ activator deficiency (variant $\mathrm{AB}$ )

$\alpha-M a n n o s i d a s e$

$\beta$-Mannosidase

$\alpha$-Fucosidase

Neuraminidase
UDP-N-acetylglycosamine: lysosomal enzyme N-acetylglucosaminyl-1phosphotransferase

Same as mucolipidosis II

Chromosome

location

$-$

-

$\begin{array}{ll}\text { 19p13.2-q12 } & \text { AF, CVB } \\ -1 \mathrm{p} 34 & \text { Poss CC } \\ \text { 4q21-qter } & \text { AF, CVB } \\ \text { 6p21.3 } & \text { AF, CVB } \\ & \text { AF, CVB } \\ \text { 6p21·3 } & \\ 20 & \text { AF, CVB } \\ & \text { AF, CVB }\end{array}$

Combined neuraminidase and

$\beta$ galactosidase deficiency

Aspartylglycosaminidase

20

Hexosaminidase $\alpha$-subunit

Hexosaminidase $\beta$-subunit

$\mathrm{Gm}_{2}$ activator protein

$15 q 22-q 25 \cdot 1$

$5 \mathrm{q} 13$

5

AF, CVB

AF, CVB

Poss CC

(v) Other lysosomal storage disorders
Type A
Type B
Type C

Farbers

Gaucher's disease:

Type 1 (non-neuronopathic)

Type 2 (acute neuronopathic)

Type 3 (Norrbottnian)

Krabbe's disease

Fabry's disease

Schindler's disease

$\mathrm{Gm}_{1}$ gangliosidosis

Wolman's disease

Cholesterol ester storage disease

Mucolipidosis type IV

(G) PEROXISOMAL DISORDERS

Zellweger syndrome

Neonatal adrenoleucodystrophy

Infantile Refsum's syndrome

(For disorders of peroxisome acyltransferase can be assayed)

Rhizomelic chondrodysplasia punctata

Pseudo-Zellweger syndrome adrenoleucodystrophy

$\mathrm{X}$ linked adrenoleucodystrophy

Refsum's disease

Acatalasia
Arylsulphatase A

Multiple lysosomal sulphatases

Sphingomyelinase

Sphingomyelinase

Cholesterol esterification

Ceramidase

Glucocerebrosidase

Glucocerebrosidase

Glucocerebrosidase

Galactocerebrosidase

$\alpha$-Galactosidase

$\alpha-\mathrm{N}$-acetylgalactosaminidase

$\beta$-Galactosidase

Acid lipase

Acid lipase

?Ganglioside sialidase
Metachromatic leucodystrophy

Multiple sulphatase deficiency

Niemann-Pick disease:

Pseudo-neonatal

$22 q 13$

17

17

lq21

lq21

lq21

14

$\mathrm{Xq} 22$

22q13-qter

$3 \mathrm{p} 21-$ cen

$10 \mathrm{q}$

$10 \mathrm{q}$

-

Peroxisome biogenesis

Peroxisome biogenesis

Peroxisome biogenesis

biogenesis a peroxisomal enzyme-dihydroxyacetone

$7 \mathrm{q} 11 \cdot 23$

AF, CVB

$\mathrm{AF}, \mathrm{CVB}$

AF, CVB

phosphate

Multiple peroxisomal enzymes -

AF, CVB

3-Oxoacyl-coenzyme A thiolase 3p23-p22

Acyl-CoA oxidase

-

Very long chain fatty acid ligase Xq28

Phytanic acid hydroxylase

$11 \mathrm{p} 13$

Catalase

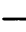

-

$\mathrm{Xq} 26-\mathrm{q} 27$

Lesch-Nyhan syndrome

Adenine phosphoribosyltransferase deficiency

Adenosine deaminase deficiency

Purine nucleoside phosphorylase deficiency
AF, CVB

Hypoxanthine phosphoribosyltransferase

Adenine phosphoribosyl- $\quad 16 \mathrm{q}$ transferase

Adenosine deaminase 20q13.1

Purine nucleoside phosphorylase $14 \mathrm{q} 13$
AF, CVB

AF, CVB

Poss CC

AF, CVB

Poss CC

UNK

AF, CVB 
Condition

Myoadenylate deaminase deficiency

Xanthinuria

Orotic aciduria

Pyrimidine-5' -nucleotidase deficiency

(I) TRACE METAL METABOLISM

Wilson's disease

Menke's disease

Haemachromatosis

Molybdenum cofactor deficiency

Isolated sulphite oxidase deficiency

(J) LIPID METABOLISM

Lipoprotein lipase deficiency

Lecithin:cholesterol acyltransferase deficiency (LCAT)

Familial hypercholesterolaemia (hyperlipidaemia type IIA)

Dysbetalipoproteinaemia (type III, hyperlipidaemia)

Tangier disease

Cerebrotendinous xanthomatosis Phytosterolaemia

(K) VITAMIN METABOLISM

Methylene tetrahydrofolate reductase deficiency

Glutamate formiminotransferase deficiency

(ii) Vitamin $B_{12}$ (cobalamin)

Transcobalamin II deficiency

Defects in adenosylcobalamin

(AdoCbl) synthesis:

cbl A mutation

cbl B mutation
Abetalipoproteinaemia

(i) Folic acid

Enzyme deficiency

Myoadenylate deaminase

Xanthine oxidase

Uridine-5'-monophosphate synthase

Pyrimidine-5'-nucleotidase

Basic defect unknown

Basic defect unknown

Molybdenum cofactor

Sulphite oxidase

Abnormal handling of apolipoprotein B

Lipoprotein lipase

Lecithin:cholesterol acyltransferase

Deficient low density lipoprotein 19 p13 $1-13 \cdot 3$ (LDL) receptors

Defective apolipoprotein E

Defective high density lipoprotein (HDL) metabolism

Mitochondrial 26-hydroxylase

Basic defect unknown

Me

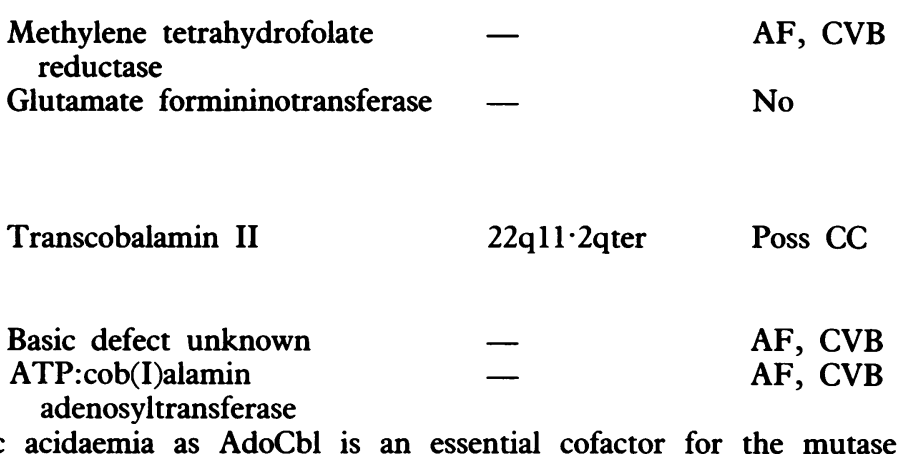

Both defects cause methylmalonic acidaemia as AdoCbl is an essential cofactor for the mutase enzyme

Defects in methylocobalamin

$(\mathrm{MeCbl})$ synthesis:

cbl E mutation

cbl G mutation

Basic defect unknown

Basic defect unknown acidaemia

cbl C mutation

cbl D mutation

cbl $\mathrm{F}$ mutation
Basic defect unknown

Basic defect unknown lysosome
Cobalamin transport from
19

AF

- $\quad$ AF

$\begin{array}{ll}\begin{array}{l}\text { Chromosome } \\ \text { location }\end{array} & \begin{array}{l}\text { Prenatal } \\ \text { diagnosis }\end{array} \\ - & \text { AF, CVB } \\ - & \text { UNK } \\ \text { 3q13 } & \text { Poss CC } \\ - & \text { UNK }\end{array}$

$\begin{array}{ll}\text { 13q14 } & \text { Poss DNA } \\ \text { Xq13 } & \text { AF, CVB } \\ 6 \mathrm{p} 21 \cdot 3 & \text { UNK } \\ - & \text { AF, CVB } \\ - & \text { Poss CC }\end{array}$

UNK

UNK

UNK

UNK

UNK

UNK

UNK

ethylmalonic

wia whout methylmalon

$\begin{array}{ll}- & \text { Poss CC } \\ - & \text { Poss CC }\end{array}$

- $\quad$ Poss CC

(L) DEFECTS IN THE SYNTHESIS AND DEGRADATION OF HAEM PROTEINS

(i) Porphyrias

$\delta$-Aminolevulinic acid dehydratase deficiency

Acute intermittent porphyria

Congenital erythropoietic porphyria

Porphyria cutanea tarda

Hereditary coproporphyria

Variegate porphyria

Erythropoietic protoporphyria

$\delta$-Aminolevulinic acid dehydratase

Porphobilinogen deaminase

Uroporphyrinogen III cosynthase

Uroporphyrindecarboxylase

Coproporphyrinogen oxidase

Protoporphyrinogen oxidase

Ferrochelatase
$9 q 34$

UNK

$\begin{array}{ll}11 \mathrm{q} 23-\mathrm{qter} & \text { AF } \\ - & \text { MET }\end{array}$

lqter-p21

9

$14 \mathrm{q} 32$

- 


\section{Condition}

Enzyme deficiency

(ii) Bilirubin metabolism

Crigler-Najiar syndrome type I

Crigler-Najiar syndrome type II

Gilbert's syndrome

Dubin-Johnson syndrome

Rotor syndrome
UDP-glucuronyltransferase

UDP-glucuronyltransferase

UDP-glucuronyltransferase

Basic defect unknown

Basic defect unknown

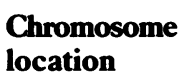

Prenatal diagnosis

$\begin{array}{ll}- & \text { No } \\ - & \text { No } \\ - & \text { No } \\ - & \text { No } \\ - & \text { No }\end{array}$

(M) DISORDERS OF MEMBRANE TRANSPORT

Cystinuria

Lysinuric protein intolerance

Hartnup disease

Cystinosis

Infantile free sialic acid storage disease

Salla disease

(N) MISCELLANEOUS DISORDERS

Lowe's syndrome

Carbonic anhydrase II

deficiency

Steroid sulphatase deficiency

Hypophosphatasia

Fumaric aciduria

Sjögren-Larsson syndrome
Renal and intestinal transport defect

Defect of cationic amino acid transport

Defect of neutral amino acid - No transport

Lysosomal cystine transport -

Lysosomal sialic acid transport

Lysosomal sialic acid transport -

Basic defect unknown

Carbonic anhydrase II

Steroid sulphatase

Alkaline phosphatase

Fumarase

Fatty alcohol NAD oxidoreductase
-

$\mathrm{Xq} 25$

$8 \mathrm{q} 22$

No

No

No

No

No

No

AF, CVB

AF, CVB

AF

UNK

No

Xpter-p22.32 AF, CVB

lp AF

Poss CC

Poss CC

$A F$ : cell free amniotic fluid or cultured amniocytes. CVB: assay performed on either uncultured or cultured villus cells. Poss CC: as far as authors' are aware prenatal diagnosis has not been performed for these disorders. However, enzyme is expressed in fibroblast cells and theoretically could be used as the basis of a prenatal test. Poss DNA: the genetic mutation causing the disoblars cells and theorically could be used as the basis of a prenatal test. Poss DNA. The genetic mutation causing the disorders has been established and may be the most appropriate approach to prenatal testing. Poss FT: the enzyme deficiency can only be detected in fetal tissue. This would usually require a fetal liver biopsy, but in some instances cocentesis. MET: metabolites from fetal urine are detected in samples obtained by amniocentesis at 14-16 weeks' gestation. UNK: as far as the authors' are aware prenatal diagnosis has not been attempted. In a number of cases the exact biochemical defect is yet to be established. ***: assay of the total activity of the pyruvate dehydrogenase complex is possible, but as far as the authors' are aware has not been applied to prenatal testing. Partial deficiencies are common leading to difficulty in interpreting results. \#\#\#: only one component of the respiratory transport chain (complex IV, cytochrome oxidase) has been studied prenatally. In families with a deletion of mitochondrial DNA a molecular approach may be more appropriate. HIST: histological changes (usually abnormal lysosomal inclusions) within amniocytes have been used as a prenatal test. UDP: uridine diphosphate. 